\title{
Poblaciones de araucaria enana (Araucaria araucana) en la Cordillera de Nahuelbuta, Chile
}

\author{
Populations of dwarf araucaria (Araucaria araucana) in the Nahuelbuta coastal range, Chile \\ Claudio Donoso a*, Mauro E. González ${ }^{a, d}$, Marco Cortés ${ }^{\text {a }}$, Conrado González ${ }^{\text {, }}$ \\ Pablo Donoso ${ }^{a, d}$, Marcelo Hernández

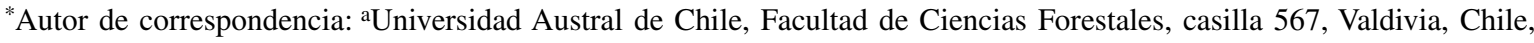 \\ tel.: 56-63-221228, fax: 56-63-221230, cdonoso@uach.cl \\ bUniversidad Católica de Temuco, Facultad de Ciencias Naturales, Temuco, Chile. \\ ${ }^{\mathrm{c} C o r p o r a c i o ́ n ~ N a c i o n a l ~ F o r e s t a l, ~ R e g i o ́ n ~ d e ~ L o s ~ R i ́ o s, ~ V a l d i v i a, ~ C h i l e . ~}$ \\ dNúcleo Científico Milenio FORECOS, Valdivia, Chile.
}

\begin{abstract}
SUMMARY
Araucaria araucana (araucaria or pehuén) can normally reach $>20 \mathrm{~m}$ in height in most sites where it grows. However, two dwarf populations of A. Araucana were found at the top of the Nahuelbuta coastal range. This study had the goal of characterizing the structure, composition, ages and chemical and physical soil properties of both populations. These populations are dominated by $A$. araucana, with Nothofagus dombeyi as the main associated species. Dwarfism is clear in both species. Individuals of A. araucana rarely exceeded $10 \mathrm{~m}$ in height with a high diameter/height relationship compared with similarly-aged normal trees. The diameter annual growth is lower than $0.12 \mathrm{~cm}$ for trees 300 years in age and basal diameters of $36 \mathrm{~cm}$. The presence of short stems and extended and fine branches, like spider legs, confirms the dwarf features of the A. araucana trees. In N. dombeyi 36 to 37 years in age the diameter annual growth is between 0.26 and $0.27 \mathrm{~cm}$, where most individuals $(60-75 \%)$ present basal diameters lower than $15 \mathrm{~cm}$ and heights between 1 and $6.5 \mathrm{~m}$. The location of these populations at the top of the Nahuelbuta coastal range over $1,000 \mathrm{~m}$ and openly exposed to the Pacific ocean results from extreme weather conditions that combined with poor and thin soils determine the low tree growth, reduced size and tortuous shapes of trees.
\end{abstract}

Key words: dwarf araucaria, structure and composition, Nothofagus dombeyi, diameter growth, age, soils.

\section{RESUMEN}

Araucaria araucana (araucaria o pehuén) desarrolla normalmente en su hábitat natural un crecimiento arbóreo con altura $>20 \mathrm{~m}$. Sin embargo, en 1984, en la cima de la Cordillera de Nahuelbuta se encontraron dos poblaciones enanas. En este trabajo se caracterizó la estructura, composición y edades de ambas poblaciones de A. araucana y Nothofagus dombeyi como especie asociada más importante, así como las características físicas y químicas de los suelos donde crecen. El enanismo se manifestó en ambas especies. En A. araucana los individuos raramente sobrepasaron $10 \mathrm{~m}$ de altura con una alta relación diámetro/altura respecto a la de árboles normales de edad similar. El crecimiento diametral anual fue $<0,12 \mathrm{~cm}$ para edades cercanas a 300 años y diámetros de base de alrededor de $36 \mathrm{~cm}$. El desarrollo de troncos cortos con ramas extendidas y delgadas, como patas de araña, refuerza el aspecto de árboles enanos. En $N$. dombeyi (edades de 36 a 37 años) el crecimiento diametral anual fue de 0,26 a 0,27 cm; la mayor parte de los individuos (60-75\%) presentó diámetro de base $<15 \mathrm{~cm}$ y altura entre 1 y $6,5 \mathrm{~m}$. La ubicación topográfica en la cima de la Cordillera de Nahuelbuta, sobre $1.000 \mathrm{~m}$ de altitud, y la exposición abierta hacia el océano Pacífico definen un clima de rigurosidad extrema que, junto con la escasa profundidad del suelo y pobreza de nutrientes, influyen en el bajo crecimiento, reducido tamaño y formas tortuosas de los árboles.

Palabras clave: araucaria enana, estructura y composición, Nothofagus dombeyi, crecimiento diametral, edad, suelos.

\section{INTRODUCCIÓN}

Araucaria araucana (Molina) K. Koch., conífera arbórea siempreverde de gran porte y longevidad, se distribuye en la Cordillera de Los Andes de Argentina y Chile, presentándose también en este último país en la
Cordillera de la Costa (Montaldo 1974, Ramírez 1978, Gajardo 1983). Actualmente se dispone de abundante información acerca de la bioecología de la especie y sus poblaciones (Montaldo 1974, Landrum y Nimlos 1975, Burns 1991), de la dinámica regenerativa en la Cordillera de Los Andes de Chile (Montaldo 1974, Schmidt 1977, 
Veblen 1982, Muñoz 1984, González 2002) y Argentina (Burns 1991, 1993), y también en la Cordillera de la Costa (Nahuelbuta) (Veblen 1982, Cortés 2003), de los suelos en que se desarrolla (Peralta 1980), y de su reproducción vegetativa (Schilling y Donoso 1976). Además se conocen antecedentes sobre algunos aspectos genecológicos y genéticos (Delmastro y Donoso 1980, Bekessy et al. 2002, 2004) y la silvicultura (Puente 1980, Schmidt et al. 1980). Una síntesis del conocimiento hasta ahora acumulado sobre la especie y los ecosistemas forestales en que participa es recopilada en González et al. (2006).

Araucaria araucana (araucaria o pehuén) presenta un amplio rango ecológico con acentuadas variaciones ambientales determinadas por cambios altitudinales y precipitaciones (Landrum y Nimlos 1975, Delmastro y Donoso 1980, Veblen 1982, González et al. 2006), así como en el tipo de suelos (Montaldo 1974, Peralta 1980, Burns 1991, Cortés et al. 2001, González 2002, Cortés 2003). En función de otras especies asociadas a A. araucana se pueden reconocer nueve subtipos señalados por Donoso (1993) sobre la base de diferentes estudios (Montaldo 1974, Landrum y Nimlos 1975, Donoso 1981b, Veblen 1982, Donoso et al. 1984, Burns 1991).

En todos los ambientes donde se desarrolla, A. araucana constituye bosques con árboles que finalmente alcanzan un buen porte. La excepción a este crecimiento son los escasos individuos achaparrados y tortuosos que crecen por sobre el límite altitudinal de la vegetación en la Cordillera de Los Andes, y que son asimilables al concepto de Krummholz (Donoso 1981a, 1993), y los grupos de A. araucana con alturas de 10 a $15 \mathrm{~m}$, fustes cortos y ramas vigorosas y marcadamente horizontales descritos por Burns (1991) en la estepa argentina, que corresponden a una condición de ecotono hacia la estepa patagónica.

Hasta 1984 las poblaciones conocidas en la Cordillera de Nahuelbuta eran descritas como rodales de árboles de A. araucana con estaturas de 20 a $25 \mathrm{~m}$, ocupando el dosel emergente y dominante. Sin embargo, en el verano de 1984 se encontraron dos poblaciones de A. araucana, con árboles pequeños, enanos, asociados a otras especies también con pequeños individuos respecto a sus tamaños normales conocidos para otros sitios. El objetivo de este trabajo fue caracterizar la estructura de estas dos poblaciones enanas de A. araucana existentes en las cimas de la Cordillera de Nahuelbuta (Donoso et al. 1984), así como dar cuenta de las condiciones de sitio donde crecen.

\section{MÉTODOS}

Área de estudio. Por la Cordillera de la Costa, A. araucana se encuentra solamente en la Cordillera de Nahuelbuta (montaña del puma en mapudungún), desde los $37^{\circ} 40^{\prime}$ a $\operatorname{los} 38^{\circ} 40^{\prime} \mathrm{S}$, y en altitudes de 800 a $1.500 \mathrm{~m}$, excepto por una población disyunta que a $600 \mathrm{~m}$ de altitud crece en el extremo sur de esta cordillera (Cortés 2003). En dicha cordillera las poblaciones enanas de A. araucana se encuentran en los predios de Caramávida ( $37^{\circ} 42^{\prime} \mathrm{S}$ y $\left.73^{\circ} 10^{\prime} \mathrm{O}\right)$ y Trongol $\left(37^{\circ} 35^{\prime}\right.$ y $\left.73^{\circ} 10^{\prime} \mathrm{O}\right)$ de la empresa Forestal Arauco, en sectores de topografía plana a ondulada, y en altitudes de 1.170 y $1.080 \mathrm{~m}$, respectivamente (figura 1).

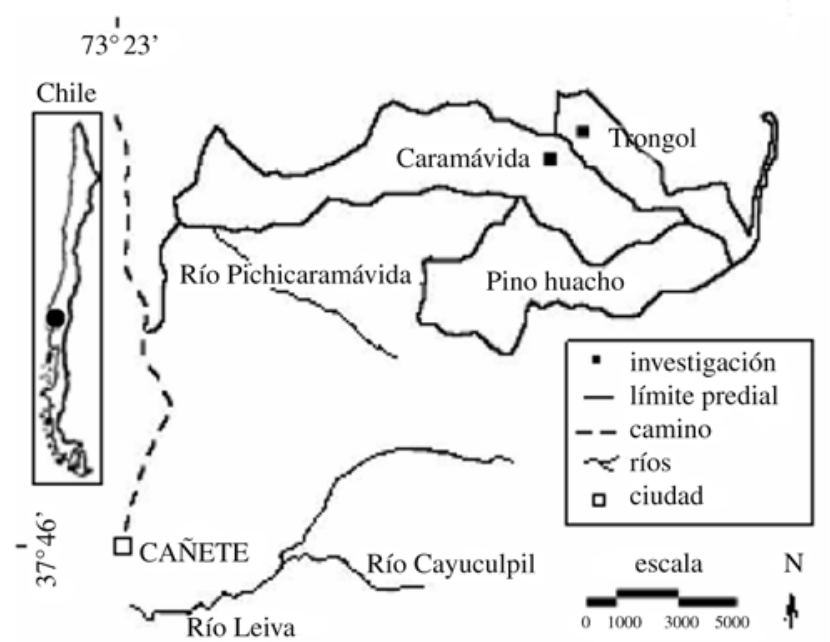

Figura 1. Plano de ubicación de las dos poblaciones de Araucaria araucana enana.

Location map of the two populations of dwarf Araucaria araucana.

El clima de la Cordillera de Nahuelbuta corresponde al tipo mediterráneo húmedo (Di Castri y Hajek 1976) con variaciones derivadas de los cambios altitudinales y de exposición. En su origen la cordillera se remonta al precámbrico con una antigüedad de más de 570 millones de años. En general los suelos derivan de material metamórfico y en algunos sectores de granito (Donoso et al. 1984). Aquellos en que crece A. araucana son delgados a moderadamente profundos, francos a arcillosos según la profundidad, con drenaje rápido a moderado, $\mathrm{pH}$ ácido de 3,0 a 4,5 y deficientes en algunos nutrientes (Donoso et al. 1984, Schlatter et al. 1995, Cortés et al. 2001).

Caracterización de las poblaciones de A. araucana enana. Las poblaciones en cada rodal se caracterizaron mediante el estudio de la estructura cuantitativa. Para determinar la frecuencia y densidad se utilizó el método de los cuartos (Cottam y Curtis 1956, Donoso 1993) con 41 puntos en Caramávida y 28 en Trongol, manteniendo una equidistancia de $20 \mathrm{~m}$. La vegetación arbustiva y herbácea se registró sólo como presencia en subparcelas de $2 \mathrm{~m}^{2}(2 \times 1), 40$ en Caramávida y 20 en Trongol.

La estructura horizontal se analizó en dos parcelas de 200 $\mathrm{m}^{2}(20 \times 10 \mathrm{~m})$ en Caramávida y de $100 \mathrm{~m}^{2}(10 \times 10 \mathrm{~m})$ en Trongol, en las cuales se midió el diámetro basal de cada individuo. Posteriormente, según registro de coordenadas se representó su posición espacial en un plano. 
Además en cada rodal, y por medio del volteo de un árbol enano de A. araucana, se obtuvieron rodelas a diferentes alturas del fuste (base, 1, 2 y $7 \mathrm{~m}$ ). Previa preparación de las muestras (Stokes y Smiley 1996) y sobre la base del conteo de los anillos de crecimiento se estimó la edad. Para una comparación, en el rodal de Trongol, el procedimiento se aplicó también en dos árboles de Nothofagus dombeyi (Mirb.) Oerst., la especie asociada de mayor frecuencia.

En cada sitio y sobre la base de sendas calicatas se realizó una descripción de los suelos. De cada horizonte se extrajo una muestra para los análisis químicos (métodos analíticos en cuadro 3), en tanto que para el análisis físico se extrajeron tres cilindros de $100 \mathrm{~cm}^{3}$.

\section{RESULTADOS}

En el predio Caramávida las especies arbóreas más abundantes en el rodal enano fueron A. araucana y Nothofagus dombeyi. Con escasa representatividad están Gevuina avellana (Mol.) y Weinmannia trichosperma (Cav.). Luego en Trongol se mantuvieron A. araucana y $N$. dombeyi como las más abundantes, seguidas de $G$. avellana. Sin embargo, aquí no se encontró $W$. trichosperma, pero sí como muy escasas Nothofagus obliqua (Mirb.) Oerst. y Embothrium coccineum (J. R. et Forster) (cuadro 1). Además, entre otras especies del sotobosque que contribuyen a la diversidad de los rodales por su mayor frecuencia estaban Griselinia scandens (R. et P.) Taub., Desfontainea spinosa (Dun.) Reiche, Chusquea spp. y Asteranthera ovata (Cav.) Hanst., seguidas de mirtáceas $y$ ericáceas arbustivas. Entre las herbáceas destacaban Quinchamalium $s p$ y gramíneas.

La densidad absoluta en árboles por hectárea fue más alta en Trongol que en Caramávida. La mayor abundancia se concentró en A. araucana, seguido de $N$. dombeyi. Con excepción de G. avellana en Trongol, las especies restantes asociadas mostraron valores de densidad insignificantes. La distancia promedio de los árboles en los rodales de la población enana de A. araucana fue menor en Trongol (2,81 m) que en Caramávida (6,5 m), confirmándose una mayor densidad y frecuencia de A. araucana para la comunidad del primero de los predios señalados. Sin embargo, en el área basal los valores promedios más altos se encontraron en Caramávida (cuadro 1). Las estructuras horizontales de los rodales de A. araucana enana también mostraron diferencias en la cobertura y los diámetros de los individuos. Es así como en Trongol se observaron las mayores coberturas y en Caramávida, aunque concentrados en pocos árboles, los más altos valores de diámetro (figura 2).

Fisonómicamente los árboles mostraron troncos cortos y ramas muy extendidas y delgadas como patas de araña (figura 3). El tamaño en altura de los individuos raramente sobrepasó los $10 \mathrm{~m}$, especialmente en Trongol, respecto a los 25 a $30 \mathrm{~m}$ de altura que pueden alcanzar en mejores sitios de la Cordillera de Nahuelbuta.
Cuadro 1. Densidad ( $\left.\mathrm{n}^{\circ} / \mathrm{ha}\right)$, frecuencia y dominancia $\left(\mathrm{m}^{2} / \mathrm{ha}\right)$ absolutas para cada especie arbórea encontrada en los rodales de Caramávida y Trongol en la Cordillera de Nahuelbuta.

Absolute density ( $\mathrm{n} / \mathrm{ha})$, frequency and dominance $\left(\mathrm{m}^{2} /\right.$ ha) for each tree species found in the Caramávida and Trongol stands in Nahuelbuta Cordillera.

\begin{tabular}{llcc}
\hline Especie & Parámetro & Caramávida & Trongol \\
\hline Araucaria & Densidad & 173 & 881 \\
& Frecuencia & 92,7 & 96,4 \\
& Dominancia & 9,7 & 25,5 \\
\hline Nothofagus & Densidad & 59 & 260 \\
dombeyi & Frecuencia & 61,0 & 46,4 \\
& Dominancia & 1,66 & 10,12 \\
\hline Gevuina & Densidad & 3 & 101 \\
avellana & Frecuencia & 4,9 & 25,0 \\
& Dominancia & 0,02 & 0,51 \\
\hline Embothrium & Densidad & - & 12 \\
coccineum & Frecuencia & - & 3,57 \\
& Dominancia & - & 0,008 \\
\hline Nothofagus & Densidad & - & 12,0 \\
obliqua & Frecuencia & - & 3,57 \\
& Dominancia & - & 0,11 \\
\hline Weinmannia & Densidad & 1 & - \\
trichosperma & Frecuencia & 2,4 & - \\
& Dominancia & 0,008 & - \\
\hline
\end{tabular}

Los valores de las edades de los individuos de A. araucana en relación con los diámetros sugieren un crecimiento relativamente lento (cuadro 2). Además, el crecimiento diametral anual a la altura del tocón fue muy similar entre los rodales con $0,119 \mathrm{~cm}$ en Caramávida y $0,120 \mathrm{~cm}$ en Trongol. Por otro lado, respecto a la A. araucana enana, el crecimiento de $N$. dombeyi fue doblemente mayor $(0,26$ a $0,27 \mathrm{~cm} / a n ̃ o)$. Sin embargo, para esta especie latifoliada tales valores son marcadamente inferiores respecto a lo observado en sitios de mejores condiciones en que muestra crecimientos de 0,5 a $0,6 \mathrm{~cm}$ anuales e incluso en plantaciones donde puede alcanzar hasta $2 \mathrm{~cm}$ anuales (Vita 1977, Donoso 1993).

En Trongol y Caramávida los suelos eran muy delgados, especialmente en el primero de los predios (cuadro 3). La densidad aparente era alta en este último sitio y media en Caramávida. El pH fue fuertemente ácido en ambos suelos y también los macronutrientes se encontraron en bajos niveles. Los contenidos de carbono y nitrógeno total fueron más altos en Caramávida que en Trongol.

\section{DISCUSIÓN Y CONCLUSIONES}

Las características de enanismo en las poblaciones de A. araucana de Caramávida y Trongol fueron claras. En Caramávida no es sorprendente que un árbol de $A$. araucana de $36,3 \mathrm{~cm}$ de diámetro en la base del tronco 

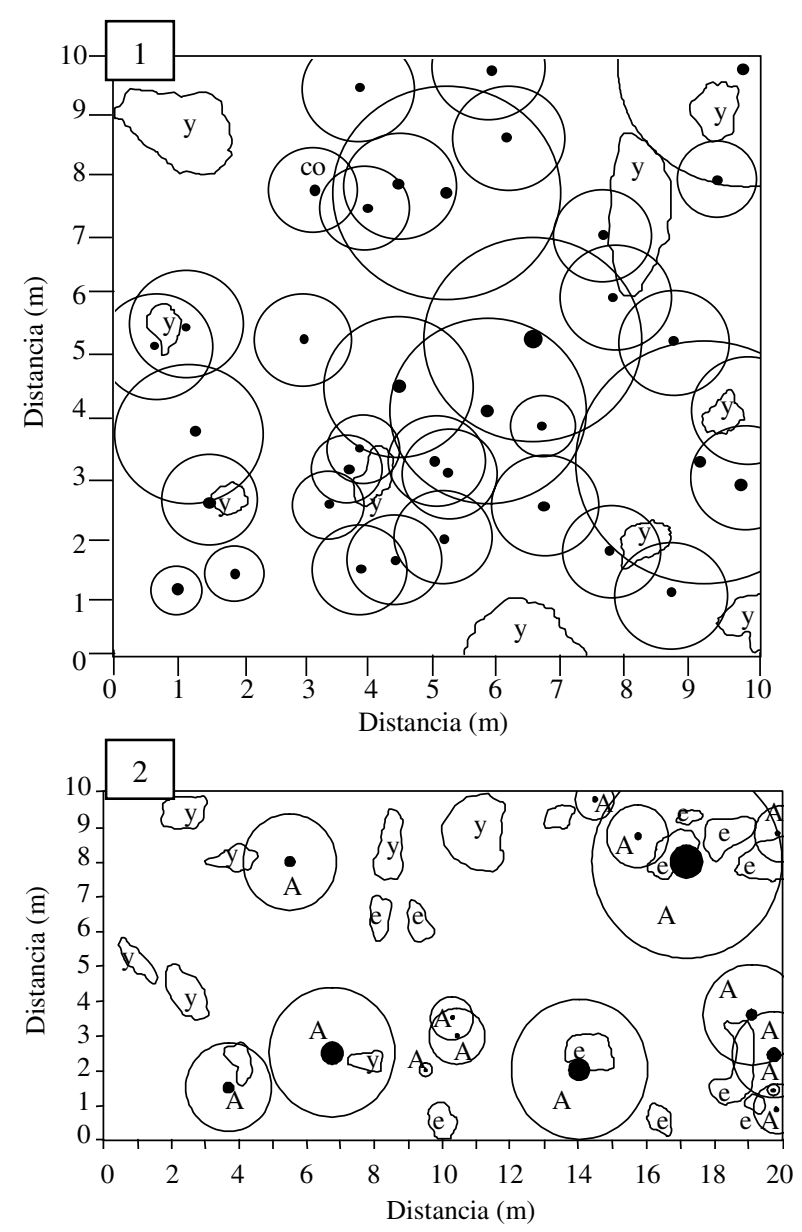

Figura 2. Estructura horizontal de dos rodales de A. araucana enana en la Cordillera de Nahuelbuta. 1) Trongol (co = Nothofagus dombeyi; y = yelmo, Griselinia sp.; círculos sin leyenda corresponden a A. araucana). 2) Caramávida ( $\mathrm{A}=$ Araucaria araucana; y = yelmo; Griselinia sp.; e = Pernettya sp.).

Horizontal structure of two dwarf Araucaria araucana stands in Cordillera of Nahuelbuta. 1) Trongol (co = Nothofagus dombeyi; $\mathrm{y}=$ Griselinia sp.; circles without labels are A. araucana trees). 2) Caramávida ( $\mathrm{A}=$ Araucaria araucana; $\mathrm{y}=$ Griselinia sp.; $\mathrm{e}=$ Pernettya $s p$.).

alcance una edad de 306 años. La relación diámetro/altura es también un claro indicador de enanismo. Este hecho se refuerza cuando otros árboles del mismo sitio, aunque de edad desconocida, alcanzan DAP entre 50 y hasta $70 \mathrm{~cm}$ para alturas de 10 a $12 \mathrm{~m}$. Del mismo modo, en Trongol el enanismo se evidencia en varios individuos cuyas alturas de 7 a $10 \mathrm{~m}$ se relacionan con diámetros de base de 50 a $60 \mathrm{~cm}$. El lento crecimiento en altura de esta especie queda además reflejado en la edad alcanzada a los 2 m de altura, con 53 años y 102 años, para Caramávida y Trongol, respectivamente. Respecto a las edades los resultados confirman lo señalado por Cortés (2003) por cuanto para la Cordillera de Nahuelbuta menciona que $A$. araucana con diámetros entre 50 y $60 \mathrm{~cm}$ puede alcanzar 200 a 300 años de edad.

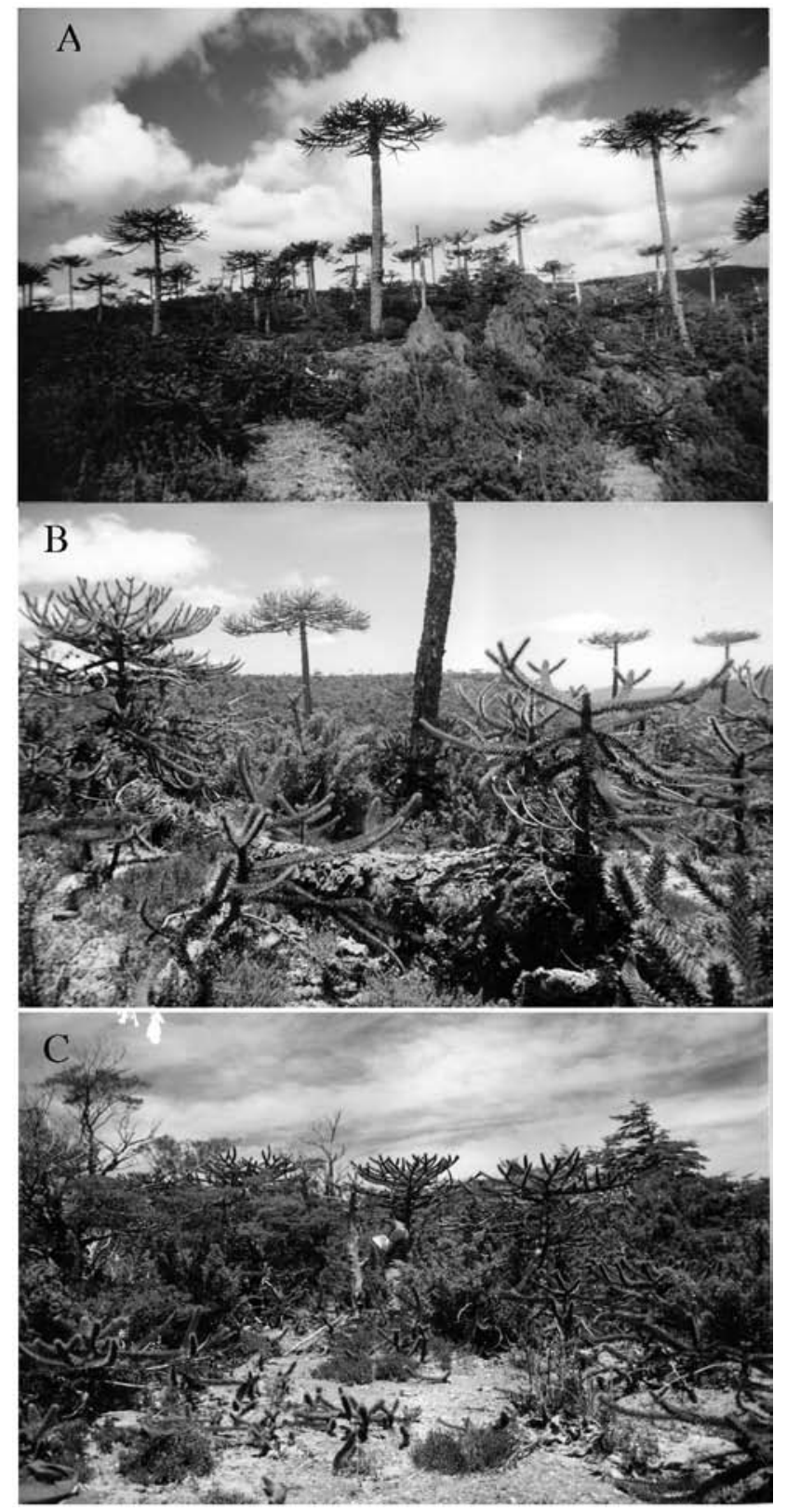

Figura 3. Sitios de estudio con Araucaria araucana enanas en Caramávida (A) y en Trongol (B y C). Nótense en estas dos últimas los troncos cortos y ramas extendidas y delgadas, como patas de araña, y el árbol de $A$. araucana caído con numerosa regeneración desde raíces.

Study sites with dwarf Araucaria araucana in Caramávida (A) and Trongol (B and C). Note for (b) and (c) the short trunks and extended and fine branches, like spider legs, and the fallen A. araucana tree with abundant regeneration from roots.

Un enanismo similar a $A$. araucana se observa en Caramávida y Trongol para $N$. dombeyi. Esta especie, en condiciones óptimas de sitios, alcanza diámetros de más de $2 \mathrm{~m}$ y alturas que sobrepasan los $40 \mathrm{~m}$. En el área de estudio, en cambio, para individuos de 36 o 37 años de edad, con diámetro de $9,7 \mathrm{~cm}$ en el tocón, el crecimiento es de $0,27 \mathrm{~cm} /$ año, inferior al crecimiento diametral de 1 
$\mathrm{cm}$ anual alcanzado en condiciones normales. La mayor parte de los árboles en ambos rodales presentaron diámetros inferiores a $15 \mathrm{~cm}$ (61\% en Trongol y 75\% en Caramávida) con alturas de 1 a $6,5 \mathrm{~m}$, mientras que sólo el $26 \%$ y el $22,5 \%$ respectivamente presentaron diámetros superiores a $20 \mathrm{~cm}$, con alturas de 7 a $14 \mathrm{~m}$.

Cuadro 2. Edades de A. araucana y $N$. dombeyi estimadas a diferentes alturas y diámetros del fuste para A. araucana y en el tocón para $N$. dombeyi.

Tree ages of $A$. araucana and $N$. dombeyi estimated at different heights and diameters in the trunk for A. araucana and in the stump for $N$. dombeyi.

\begin{tabular}{|c|c|c|c|}
\hline $\begin{array}{l}\text { Altura de la } \\
\text { rodela }\end{array}$ & $\begin{array}{l}\text { Edad } \\
\text { (años) }\end{array}$ & $\begin{array}{l}\text { Diámetro } \\
(\mathrm{cm})\end{array}$ & $\begin{array}{c}\text { Espesor de } \\
\text { corteza }(\mathrm{cm})\end{array}$ \\
\hline \multicolumn{4}{|c|}{ Araucaria araucana enana de Caramávida } \\
\hline Tocón & 306 & 36,3 & 5,5 \\
\hline $2 \mathrm{~m}$ & 253 & 30,2 & 3,9 \\
\hline $7 \mathrm{~m}$ & 117 & 19,2 & 2,8 \\
\hline \multicolumn{4}{|c|}{ Araucaria araucana enana de Trongol } \\
\hline Tocón & 143 & 17,3 & 3,5 \\
\hline $1 \mathrm{~m}$ & 85 & 15,5 & 2,8 \\
\hline $2 \mathrm{~m}$ & 41 & 11,2 & 1,9 \\
\hline \multicolumn{4}{|c|}{ Nothofagus dombeyi de Trongol } \\
\hline Tocón árbol 1 & 37 & 9,7 & 0,4 \\
\hline Tocón árbol 2 & 36 & 9,7 & 0,4 \\
\hline
\end{tabular}

Cuadro 3. Propiedades físicas y químicas de los suelos con Araucaria enana en Caramávida y Trongol, Cordillera de Nahuelbuta.

Physical and chemical soil properties for dwarf Araucaria in Caramávida and Trongol, Cordillera de Nahuelbuta.

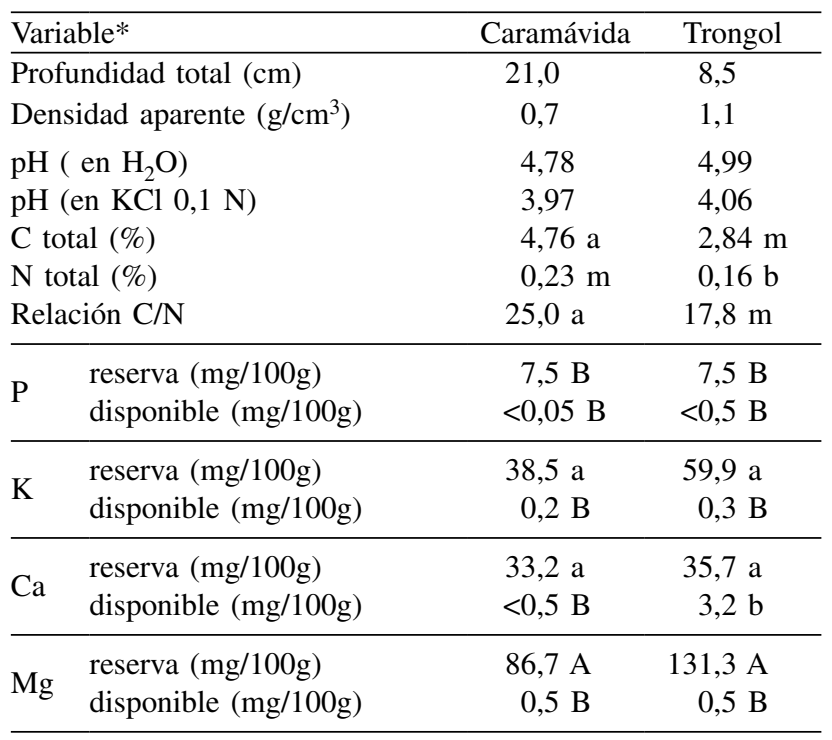

$\mathrm{C}$ total: oxidación húmeda; $\mathrm{N}$ total: método Kjeldahl; reserva de elementos: extracto en $\mathrm{HCl} 3 \%$; elementos disponibles: extracto en ácido cítrico $1 \%$. $\mathrm{B}=$ muy bajo $\mathrm{b}=$ bajo; $\mathrm{m}=$ moderado $; \mathrm{a}=$ alto; $\mathrm{A}=$ muy alto.
Los suelos son pobres en nutrientes y con escasa profundidad. Además, la ubicación topográfica en la cima de la montaña y por sobre los $1.000 \mathrm{~m}$ de altitud y con abierta exposición a los vientos del noroeste influyen en la rigurosidad del clima con fuertes vientos, altas temperaturas y sequía en verano, que en invierno descienden con frecuente precipitación nivosa. Sin duda, estas condiciones ambientales inciden en el bajo crecimiento, reducido tamaño y formas tortuosas de los árboles. La reducida profundidad del suelo, en Trongol por ejemplo, sugiere ser la causa de la intensa caída de árboles. Por otra parte, la mayor cobertura con árboles de menor tamaño obedecería también a la abundante reproducción vegetativa desde raíces y de retoños de vástagos de troncos caídos que se mantienen enraizados (figura 3B y 3C).

El fenómeno de poblaciones de bosques enanos se ha reportado también para otras especies y sitios del mundo, como Pinus rigida Mill. en Mt. Everett en Massachusetts (Motzkin et al. 2002) y Pinus culminicola Andersen. et Beaman en Cerro Potosí, Sierra Maestra Oriental en Nuevo México (Jiménez et al. 1996). En Chile, igualmente se han descrito algunas poblaciones enanas de alerce (Fitzroya cupressoides (Mol) Johnston). En la Cordillera de la Costa al sur de Valdivia $\left(\sim 40^{\circ} \mathrm{S}\right)$ y en las cumbres de la cordillera Pelada, bosques de alerce y coihue de Magallanes (Nothofagus betuloides (Mirb.) Oerst.) exhiben también una estructura y fisonomía de enanismo (Veblen y Ashton 1982).

Para A. araucana esta característica de enanismo sería el resultado tanto de condiciones impuestas por suelos de origen metamórfico pobres y muy delgados con baja disponibilidad de agua en verano, así como por las extremas características climáticas con nieve y bajas temperaturas en invierno.

\section{AGRADECIMIENTOS}

Los autores agradecen a la Empresa Forestal Arauco que con apoyo del proyecto "Antecedentes para el manejo del bosque nativo de Forestal Arauco en la Cordillera de Nahuelbuta" hizo posible tomar la información de terreno, y al Sr. Jorge Silva, estudiante de Ingeniería Forestal, por la edición y mejoramiento de las figuras.

\section{REFERENCIAS}

Bekessy SA, TR Alnutt, AC Premoli, A Lara, RA Ennos, MA Burgnam, M Cortés, AC Newton. 2002. Genetic variation in the vulnerable and endemic Monkey Puzzle tree, detected using RAPDs. Heredity 88: 1-7.

Bekessy SA, A Lara, ME González, M Cortés, L Gallo, AC Premoli, AC Newton. 2004. Variación en Araucaria araucana (Molina) K. Koch (Araucaria o Pehuén). In Donoso 
C, A Premoli, L Gallo, R Ipinza eds. Variación intraespecífica en las especies arbóreas de los bosques templados de Chile y Argentina. Editorial Universitaria, Santiago, Chile. p. 215-231.

Burns BR. 1991. Regeneration dynamics of Araucaria. PhD thesis. University of Colorado, Department of Geography, Boulder, USA. 211 p.

Burns BR. 1993. Fire-induced dynamics of Araucaria araucanaNothofagus antarctica forest in the southern Andes. Journal of Biogeography 20: 669-685.

Cortés M. 2003. Dinámica y conservación de Araucaria araucana (Mol.) Koch en la Cordillera de la Costa de Chile. Tesis de Magister. Valdivia, Chile. Facultad de Ciencias Forestales, Universidad Austral de Chile. 112 p.

Cortés M, V Gerding, O Thiers. 2001. Caracterización de la fertilidad de dos sitios con Araucaria araucana (Mol.) Koch en la Cordillera de la Costa de Chile. XIII Reunión Anual de la Sociedad de Botánica de Chile. La Serena, Chile. Gayana Botánica 58 (1): 73.

Cottam G, JT Curtis. 1956. The use of distance measures in phytosociological sampling. Ecology 37: 451-460.

Delmastro R, C Donoso. 1980. Review of distribution, variation and utilization of gene resources of Araucaria araucana (Mol.) Koch in Chile. Simposio IUFRO en melhoramiento genético e productividade de espécies florestais de rápido crescimento, Brazil.

Di Castri F, E Hajek. 1976. Bioclimatología de Chile. Vicerrectoría Académica de la Universidad Católica de Chile. Santiago, Chile. 129 p.

Donoso C. 1981a. Ecología forestal. El bosque y su medioambiente. Editorial Universitaria. Santiago, Chile. 369 p.

Donoso C. 1981b. Tipos forestales de los bosques nativos de Chile. Documento de Trabajo $\mathrm{N}^{\circ} 38$. Investigación y desarrollo forestal (CONAF, PNUD, FAO) (Publicación FAO Chile). 70 p.

Donoso C. 1993. Bosques templados de Chile y Argentina. Variación, estructura y dinámica. Ecología Forestal. Editorial Universitaria, Santiago, Chile. 484 p.

Donoso C, V Gerding, B Olivares, P Real, V Sandoval, R Schlatter, F Schlegel. 1984. Antecedentes para el manejo del bosque nativo de Forestal Arauco. Universidad Austral de Chile. Informe de Convenio $\mathrm{N}^{\circ} 74.183 \mathrm{p}$.

Gajardo R. 1983. Sistema básico de la clasificación de la vegetación nativa chilena. Universidad de Chile. Departamento de Silvicultura y Manejo/ Corporación Nacional Forestal. $165 \mathrm{p}$.

González ME. 2002. Fire history of Araucaria-Nothofagus forests in the Andean cordillera of south-central Chile. PhD. Thesis. University of Colorado. Boulder, USA. 158 p.

González ME, M Cortés, F Izquierdo, L Gallo, C Echeverría, S Bekessy, P Montaldo. 2006. Araucaria araucana (Molina) K. Koch. Araucaria (o), Pehuén, Pino piñonero, Pino de Neuquén, Monkey Puzzle Tree. In Donoso C ed. Las especies arbóreas de los bosques templados de Chile y Argentina. Autoecología. Marisa Cuneo Ediciones. Valdivia, Chile. p. 36-53.
Jiménez J, H Kramer, O Aguirre. 1996. Pinus culmicola: discovery and preservation of a Mexican dwarf pine. Forst-und-Holz 51: 20, 664-665.

Landrum L, TJ Nimlos. 1975. Gradientes florales y morfología asociada del suelo en la Reserva Forestal de Malalcahuello, Chile. Boletín Técnico $\mathrm{N}^{\circ} 35$. Facultad de Ciencias Forestales Universidad de Chile, Santiago, Chile. 59 p.

Montaldo P. 1974. La bioecología de Araucaria araucana (Mol.) Koch. Instituto Forestal Latinoamericano de Investigación y Capacitación. Boletín 46: 3-55.

Motzkin G, DA Orwig, DR Foster. 2002. Vegetation and disturbance history of a rare dwarf pitch pine community in western New England, USA. Journal of Biogeography 29: $10-11$.

Muñoz IR. 1984. Análisis de la productividad de semillas de Araucaria araucana (Mol.) Koch en el área de Lonquimay, IX Región. Tesis Universidad de Chile, Santiago, Chile. $140 \mathrm{p}$.

Peralta M. 1980. Geomorfología, clima y suelos del tipo forestal Araucaria en Lonquimay. Boletín Técnico $\mathrm{N}^{\circ}$ 57. Facultad de Ciencias Forestales, Universidad de Chile. Santiago, Chile. 15 p.

Puente M. 1980. Utilización de un bosque de tipo Araucaria con criterios de permanencia. Boletín Técnico $\mathrm{N}^{\circ}$ 57. Facultad de Ciencias Forestales, Universidad de Chile. Santiago, Chile. 22 p.

Ramírez C. 1978. Estudio florístico y vegetacional del Parque Nacional Tolhuaca. (Malleco, Chile). Museo Nacional de Historia Natural de Santiago, Chile. Publicación ocasional 24: 3-23.

Schilling G, C Donoso. 1976. Reproducción vegetativa natural de Araucaria araucana (Mol.) Koch. Investigación Agrícola 2(3): 121-122.

Schlatter J, V Gerding, J Adriazola. 1995. Sistema de ordenamiento de la tierra: herramienta para la planificación forestal aplicada a las regiones VII, VIII y IX. Valdivia, Chile. Universidad Austral de Chile. 90 p.

Schmidt H. 1977. Dinámica de un bosque virgen de AraucariaLenga. Bosque 2: 3-11.

Schmidt H, MM Toral, P Burgos. 1980. Aspectos de estructura y de regeneración natural para el manejo silvícola de los bosques de Araucaria-Lenga de Chile. Forestry problems of the Genus Araucaria. Proceedings of IUFRO meeting held in Curitiba, Paraná, Brazil. pp. 159-166.

Stokes MA, TL Smiley. 1996. An introduction to tree-ring dating. The University Arizona Press, Tucson, Arizona, USA. 73 p.

Veblen TT. 1982. Regeneration patterns of Araucaria araucana forests in Chile. Journal of Biogeography 9: 11-28.

Veblen TT, D Ashton. 1982. The regeneration status of Fitzroya cupressoides in the Cordillera Pelada, Chile. Biological Conservation 23: 141-161.

Vita A. 1977. Crecimiento de algunas especies forestales en el arboretum del Centro Experimental de Frutillar, Décima Región. Boletín Técnico $\mathrm{N}^{\circ}$ 47. Facultad de Ciencias Forestales, Universidad de Chile. 16 p. 


\section{INSTRUCCIONES PARA LOS AUTORES DE LA REVISTA BOSQUE}

BOSQUE publica trabajos originales relacionados con el manejo y producción de recursos forestales, ciencias y tecnología de la madera, silvicultura, ecología forestal, conservación de recursos naturales y desarrollo rural asociados con los ecosistemas forestales. Las contribuciones podrán ser en las modalidades de artículos, notas u opiniones, en castellano o inglés.

- Artículos. Informan acerca de investigaciones inéditas de carácter científico que proyectan el conocimiento actualizado en un campo particular contemplado en los ámbitos de la revista y están sustentados en datos procedimentales propios o generados a partir de otros estudios publicados. La extensión máxima de los manuscritos será de 30 páginas u 8.000 palabras, incluyendo resumen, cuadros, figuras y referencias bibliográficas.

- Opiniones. Analizan, desde un punto de vista personal o con apoyo bibliográfico, un tema de actualidad relacionado con el carácter de la revista, o bien analizan y critican publicaciones de actualidad. La extensión máxima de los manuscritos será de 10 páginas o 3.000 palabras, incluyendo resumen, cuadros, figuras y referencias bibliográficas.

- Notas. Describen metodologías o técnicas nuevas en el ámbito de la revista, o bien informan acerca de investigaciones en desarrollo, con resultados preliminares. La extensión máxima de los manuscritos será de 10 páginas o 3.000 palabras, incluyendo resumen, cuadros, figuras y referencias bibliográficas.

\section{Carta de envío}

Los autores deberán acompañar su manuscrito con una carta de envío que indique que el trabajo es original, no ha sido publicado previamente y no está siendo considerado para publicación en otro medio de difusión. Se deberá señalar el tipo de contribución del manuscrito (Artículo, Opinión, Nota). La carta deberá ser firmada al menos por el autor líder del manuscrito.

\section{Estructura de las contribuciones}

La organización de Artículos y Notas debe seguir la siguiente estructura:

- Título: Elegir con mucho cuidado todas las palabras del título; su asociación con otras palabras debería ser cuidadosamente revisada. Debido al acceso internacional de la revista, se recomienda incluir en el título información relevante sobre la localización geográfica del estudio.

- Autores: indicar el nombre y apellido de todos los autores con letras minúsculas, con las letras iniciales en mayúscula. La dirección de correo electrónico y las instituciones a las cuales pertenecen deberán indicarse como nota usando una letra en superíndice. Además, incluir la dirección, teléfono y fax del autor receptor de correspondencia. Éste deberá ser identificado con un asterisco en superíndice $(*)$.

- Resumen: debe contener el objetivo, fundamentos metodológicos, resultados y conclusiones más relevantes, con un máximo de 250 palabras. Evite descripciones largas de métodos y no incluya citas bibliográficas.
- Palabras clave: como máximo cinco palabras (puede incluir una o dos frases breves de un máximo de tres palabras) que identifiquen claramente el tema del trabajo. Se sugiere usar nuevas palabras no incluidas en el título del manuscrito.

- Introducción: comprende planteamiento del problema, importancia del tema, hipótesis si compete, objetivos, alcances del trabajo y limitaciones para su desarrollo, si es que las hubo. En este capítulo se realizará una síntesis e interpretación de la literatura relacionada directamente con el título y objetivos del trabajo.

- Métodos: proveerá información suficiente y concisa de manera que el problema o experimento pueda ser reproducido o fácilmente entendido por especialistas en la materia. Deberán señalarse claramente las especificaciones técnicas y procedencia de los materiales usados, sin describir materiales triviales. Los organismos bióticos deberán ser convenientemente identificados de acuerdo con las normas internacionales que correspondan. En los métodos empleados se deberá señalar claramente el procedimiento experimental o de captación de datos y los métodos estadísticos, así como los programas computacionales. Si el método no fuese original, se indicará bibliográficamente. Si fuera original o modificado se describirá convenientemente. En cualquier caso, la presentación de varios métodos será cronológica.

- Resultados: incluye la presentación sintética, ordenada y elaborada de la información obtenida. Entrega resultados en forma escrita con apoyo de cuadros y figuras, si corresponde, conjuntamente con análisis e interpretación de los datos. Se deberá evitar tanto la repetición de detalles dados en otros capítulos como la descripción de aquello que sea evidente al examinar los cuadros o figuras que se presenten.

- Discusión: incluye la interpretación integrada de los resultados y, cuando corresponda, la comparación de ellos con los de publicaciones previas. Es un análisis crítico de los resultados de acuerdo con los objetivos y la hipótesis, si fuera el caso. Debe comentarse el significado y la validez de los resultados, de acuerdo con los alcances definidos para el trabajo y los métodos aplicados. En este capítulo no deberán repetirse los resultados obtenidos.

- Conclusiones: podrán ser incluidas en un capítulo único de Conclusiones, o bien integradas en Discusión. En caso de presentarlas como un capítulo, se incluirán allí en forma precisa y concisa aquellas ideas más relevantes que se deriven directamente de lo entregado por el trabajo. Deben dar respuesta a las hipótesis y/o a los objetivos planteados en la Introducción. Deben redactarse en forma clara y objetiva sin incluir citas bibliográficas. Pueden incluir recomendaciones para trabajos futuros.

- Agradecimientos: de ser necesario, se podrán incluir en este acápite a personas o instituciones, en una muy breve mención, que contribuyeron con financiamiento u otro tipo de colaboración.

- Referencias: se indicarán las referencias de todas las citas bibliográficas señaladas en el texto, ordenadas alfabéticamente. La precisión y veracidad de los datos entregados en las referencias bibliográficas, son responsabilidad del o los autores de las contribuciones y deben corresponder a publicaciones originales.

Para las Opiniones no se exige seguir la estructura indicada anteriormente. 


\section{Estilo y Formato}

Use tiempo presente cuando se refiera a resultados publicados previamente. Esto ayuda a diferenciar entre los hallazgos de su estudio y los hallazgos de otros estudios. En general, el Resumen, Métodos y Resultados de su manuscrito deberían estar en tiempo pasado, y la Introducción y Discusión en tiempo presente.

Trabajo escrito en hojas tamaño carta $(279 \times 216 \mathrm{~mm})$, márgenes de $2 \mathrm{~cm}$ por lado, interlineado a espacio y medio, letra Times New Roman, tamaño 12 puntos, con numeración de página en el extremo inferior derecho y número de línea correlativo para todo el trabajo, a la izquierda. Separar los párrafos a renglón seguido y con sangría de ocho caracteres a la izquierda de la primera línea. Debe presentarse en archivos electrónicos con procesador de texto Word o formato RTF (no se aceptan trabajos en formato PDF).

El título principal con letras minúsculas y negritas, centrado. En él deberá omitirse la mención de los autores de nombres científicos, los que, sin embargo, se presentarán la primera vez que se mencionen en el texto. En el encabezado superior derecho de cada página debe incluirse un título abreviado con un máximo de 40 caracteres y espacios.

Las ecuaciones se numerarán en el margen derecho con paréntesis cuadrados "[ ]"; en el texto se mencionarán de acuerdo con esta numeración.

Las unidades de medidas deberán circunscribirse al Sistema Internacional (SI). En la notación numérica, los decimales deberán ser separados por coma (,) y las unidades de miles por punto (.). En los textos en inglés, los decimales separados por punto y las unidades de miles por coma. Usar cero al comienzo de números menores a una unidad, incluyendo valores de probabilidad (por ejemplo, $P<0,001)$.

La descripción de los resultados de cada prueba estadística en el texto debe incluir el valor exacto de probabilidad asociado $P$. Para valores de $P$ menores que 0,001 , indique como $P<0,001$. En cuadros y figuras usar asteriscos para señalar el nivel de significancia de las pruebas estadísticas: $*=P<0,05 ; * *=P$ $<0,01 ; * * *=P<0,001 ; \mathrm{ns}=$ no significativo

Debe indicarse el nombre científico de todos los organismos biológicos que aparezcan en el texto, de acuerdo con la nomenclatura internacional respectiva. Si un nombre común es usado para una especie, la primera vez que se cite en el texto se debe dar a continuación su nombre científico en cursiva entre paréntesis, por ejemplo, coihue (Nothofagus dombeyi). Citas posteriores pueden aparecer con el nombre del género abreviado seguido del adjetivo del nombre científico, por ejemplo, lenga ( $N$. pumilio), siempre y cuando no produzca confusiones con otras especies citadas en el manuscrito.

En los cuadros se deben incluir los datos alfanuméricos ordenados en filas y columnas. Sólo los encabezamientos de las columnas y los títulos generales se separan con líneas horizontales; las columnas de datos deben separarse por espacios y no por líneas verticales. En las figuras se incluyen otras formas de presentación de datos o información, como gráficos, dibujos, fotografías y mapas. En cuadros y figuras se deben incluir los títulos autoexplicativos en castellano e inglés numerados en forma consecutiva (cuadro 1 ., cuadro 2 ., ...; figura 1 ., figura $2 ., \ldots$ ). Las figuras llevan el título en el margen inferior y los cuadros en el margen superior. Los cuadros y figuras deben tener una resolución tal que permitan ser reducidos sin perder legibilidad. Sólo se trabaja en blanco, negro y tonos de grises. La inclu- sión de figuras con colores será de costo del autor y para ello deberá comunicarse previamente con el Editor. El espacio que estos ocupen en el trabajo deberá ser menor al 50\% del total del impreso. Dentro del texto se debe indicar claramente la ubicación de figuras y cuadros, los que deben ser anexados en archivos separados.

Los manuscritos en castellano deben incluir en un archivo separado las respectivas traducciones al inglés de:

- Título del manuscrito.

- Summary: debe ser equivalente en contenido al resumen en castellano.

- Key words: equivalentes a las palabras clave en castellano.

- Títulos de cuadros y de figuras.

En el caso de manuscritos en inglés, se debe incluir el respectivo texto en castellano.

\section{Envío de documentos}

Se solicita etiquetar adecuadamente los archivos digitales del manuscrito a enviar por los autores. Los archivos deberán ser nombrados según el apellido del autor líder o de correspondencia, seguida del tipo de información contenida en el archivo. Por ejemplo, los archivos digitales del manuscrito liderado por el autor Pedro López, se etiquetarán de la siguiente forma:

Lopez_texto.doc: Texto principal del trabajo.

Lopez_cuadros.doc: Cuadros con sus títulos en castellano.

Lopez_figuras.doc: Figuras con sus títulos en castellano.

Lopez_ingles.doc: Textos en inglés con el siguiente orden: título del trabajo, summary, key words, títulos de cuadros y de figuras.

\section{Citas y Referencias}

Las citas bibliográficas se indicarán en el texto por el apellido del o los autores, seguido del año de publicación. Algunos ejemplos de citas bibliográficas más frecuentes son:

\section{Citas bibliográficas de uno y dos autores:}

Santamaría (2001) constata que el crecimiento .........

.... están influidos por el sitio en cuestión (Santamaría 2001, López y Castro 2004).

Citas bibliográficas de más de dos autores:

Barría et al. (1999) señalan como factor más importante ....... entre otros, el diámetro y la altura (Barría et al. 1999, Morán et al. 2002).

Citas bibliográficas de un mismo autor, publicadas en un mismo año:

Rodríguez (1997abd) observa que en cada unidad de muestreo ........

....... lo que es coincidente con estudios anteriores (Rodríguez 1997ab, Morán et al. 2003acd).

Citas de más de una publicación a la vez, se ordenan cronológicamente:

Cerón (1980), García y Villanueva (1994) y Suárez et al. (2001) al analizar los componentes edafoclimáticos...

En el capítulo Referencias, las referencias bibliográficas deben incluir el apellido paterno e iniciales del o los nombres de 
todos los autores, el año de publicación, el título y la información complementaria que permita localizar la fuente del documento en cuestión. Algunos ejemplos de referencias bibliográficas más frecuentes son:

Referencias de artículos en revistas periódicas:

Guddants S. 1998. Replicating sawmill sawing with topsan using C.T. images of a full-length hardwood log. Forest Products Journal 48(1):72-75.

Keyes M, C Grieg. 1981. Above and below-ground biomass net production in 40-year-old-Douglas-fir stand on low and high productivity sites. Can. J. For. Res. 11:599-605.

Karzulovic JT, MI Dinator, J Morales, V Gaete, A Barrios. 2005. Determinación del diámetro del cilindro central defectuoso en trozas podadas de pino radiata (Pinus radiata) mediante atenuación de radiación gamma. Bosque 26(1):109-122.

Referencias de libros como un todo:

Morales EH. 2005. Diseño experimental a través del Análisis de Varianza y Modelo de Regresión Lineal. Santiago, Chile. Andros. 248 p.

CONAF (Corporación Nacional Forestal, CL). 1997. Estadísticas de visitantes e ingresos propios de Áreas Silvestres Protegidas de la Décima Región de Los Lagos. 52 p. (Informe Estadístico No 47).

Referencias a partes o capítulos de libros:

Gutiérrez B, R Ipinza. 2000. Evaluación de parámetros genéticos en Nothofagus. In Ipinza R, B Gutiérrez, V Emhart eds. Domesticación y Mejora Genética de Raulí y Roble. Valdivia, Chile. Exion. p. 371-390.

Referencias a Memorias, Tesis, Seminarios de Titulación o Trabajos de Titulación:

Emhart V. 1996. Diseño y Establecimiento de un Huerto Semillero Clonal de Eucalyptus nitens (Deane et Maiden) con fines de Producción, Investigación y Docencia. Tesis Ingeniero Forestal. Valdivia, Chile. Facultad de Ciencias Forestales, Universidad Austral de Chile. 79 p.

Aparicio J. 2001. Rendimiento y Biomasa de Eucalyptus nitens con alternativas nutricionales para una silvicultura sustentable en suelo rojo arcilloso. Tesis Magíster en Ciencias. Valdivia, Chile. Facultad de Ciencias Forestales, Universidad Austral de Chile. 234 p.

\section{Referencias a documentos en Internet:}

DeAngelis JD. 1999. European Pine Shoot Moth. Oregon State University Extension (Urban Entomology Notes). Consultado 10 jul. 2005. Disponible en http://www.ent.orst.edu/urban/home. html.

Para mayor información respecto de otros casos específicos relacionados con las citas bibliográficas y referencias bibliográficas se pueden consultar los documentos que a continuación se señalan. No obstante, el orden y la tipografía de los elementos constituyentes de las citas y referencias bibliográficas deberán ajustarse a la reglamentación de la revista BOSQUE.

Redacción de Referencias Bibliográficas: Normas Técnicas del IICA y CATIE. Consultado 21 octubre 2005. Disponible en http:// orton.catie.ac.cr/bco/normas de redaccion.html

The Council of Biology Editors (CBE). 1994. Scientific Style and Format: The CBE Manual for Authors, Editors, and Publishers. 6 ed. Cambridge, New York. Cambridge University Press. 704 p.

\section{Proceso de publicación}

Las contribuciones se recibirán por correo electrónico en la dirección revistabosque@uach.cl o impreso por triplicado más el disco con los respectivos archivos en oficina de BOSQUE (Revista BOSQUE, Casilla 567, Valdivia, Chile); el autor de correspondencia recibirá una carta de acuse de recibo del Editor.

Una vez que el Comité Editor ha decidido enviar el manuscrito a arbitraje, las observaciones de los árbitros serán evaluadas por el Comité Editor, el cual informará a los autores de la decisión de continuación en el proceso de publicación. En el caso de que el manuscrito sea aceptado, el Comité Editor enviará al autor de correspondencia una carta de aceptación de su manuscrito, indicando el tipo de modificación necesaria. En no más de tres semanas el autor de correspondencia deberá devolver una versión modificada (por correo electrónico) a la revista, para que el Comité Editor analice nuevamente el manuscrito. Una contribución puede ser rechazada por el Comité Editor en cualquiera de las instancias del proceso de publicación, ya sea por cuestiones de fondo o de forma.

Para cualquier duda se sugiere revisar la información de nuestra página web www.facultadforestal.cl/revista_bosque o en www.scielo.cl, o contactarse con el Editor.

A partir de 2003 la revista BOSQUE es parte de SciELOChile, donde podrá encontrar la versión electrónica de sus contribuciones: www.scielo.cl 